\title{
Early-onset Purkinje cell dysfunction underlies cerebellar ataxia in peroxisomal multifunctional protein-2 deficiency
}

Stephanie De Munter ${ }^{1}$, Simon Verheijden ${ }^{2}$, Esther Vanderstuyft ${ }^{1}$, Ana Rita Malheiro ${ }^{3}$, Pedro Brites $^{3}$, David Gall ${ }^{4}$, Serge N. Schiffmann ${ }^{4}$, and Myriam Baes ${ }^{1}$

${ }^{I}$ KU Leuven - University of Leuven, Department of Pharmaceutical and Pharmacological Sciences, Cell Metabolism, B-3000 Leuven, Belgium, ${ }^{2}$ KU Leuven - University of Leuven, Department of Clinical and Experimental Medicine, TARGID, B-3000 Leuven, Belgium; ${ }^{3}$ Instituto de Biologia Molecular e Celular - IBMC and Instituto de Inovação e Investigação em Saúde, University of Porto, Nerve Regeneration group, 4200-135 Porto, Portugal. ${ }^{4}$ ULB - Faculté de Médecine - Campus Erasme - Laboratory of Neurophysiology, ULB-Neuroscience Institute, B-1070 Brussels, Belgium.

e-mail addresses:

Stephanie.Demunter@pharm.kuleuven.be

Simon.Verheijden@med.kuleuven.be

Esther.Vanderstuvft@gmail.com

Ana.malheiro@ibmc.up.pt

Dgall@ulb.ac.be

Sschiffm@ulb.ac.be

Pedro.Brites@ibmc.up.pt

Myriam.Baes@pharm.kuleuven.be

Corresponding author:

Myriam Baes, $\mathrm{PhD}$

Laboratory for Cell Metabolism

Faculty of Pharmaceutical and Pharmacological Sciences

Campus Gasthuisberg O/N2

Herestraat 49

B 3000 Leuven

$\mathrm{Tel}+3216330853$

Fax +3216330856 


\section{ABSTRACT}

The cerebellar pathologies in peroxisomal diseases underscore that these organelles are required for the normal development and maintenance of the cerebellum, but the mechanisms have not been resolved. Here we investigated the origins of the early-onset coordination impairment in a mouse model with neural selective deficiency of multifunctional protein-2, the central enzyme of peroxisomal $\beta$-oxidation. At the age of 4 weeks, Nestin-Mfp $2^{-/}$mice showed impaired motor learning on the accelerating rotarod and underperformed on the balance beam test. The gross morphology of the cerebellum and Purkinje cell arborization were normal. However, electrophysiology revealed a reduced Purkinje cell firing rate, a decreased excitability and an increased membrane capacitance. The distribution of climbing and parallel fiber synapses on Purkinje cells was immature and was accompanied by an increased spine length. Despite normal myelination, Purkinje cell axon degeneration was evident from the occurrence of axonal swellings containing accumulated organelles. In conclusion, the electrical activity, axonal integrity and wiring of Purkinje cells are exquisitely dependent on intact peroxisomal $\beta$-oxidation in neural cells.

\section{KEYWORDS}

Peroxisomes, $\beta$-oxidation, multifunctional protein-2, Purkinje cell, ataxia, axonal swellings, electrophysiology, climbing fiber, parallel fiber.

\section{ABBREVIATIONS}

APP: anti-Alzheimer precursor protein; BOS: base of support; CF: climbing fiber; DAPI: 4',6diamidino-2-phenylindole; ECL: enhanced chemiluminescence; EGL: external granule cell layer; GABA: gamma-aminobutyric acid; GluR $\delta 2$ : glutamate receptor $\delta 2$ subunit; H\&E: haematoxylin and eosin; HRP: horseradish peroxidase; HSD17B4: 17ß-hydroxysteroid dehydrogenase type 4; IGL: internal granule cell layer; LF: left front; LH: left hind; MFP2: multifunctional protein-2; ML: molecular layer; P: postnatal day; PBD: peroxisomal biogenesis disorders; PBS: phosphate buffered saline; PC: Purkinje cell; PF: parallel fiber; PFA: paraformaldehyde; PPD: p-phenylenediamine; PUFA: polyunsaturated fatty acids; RF: right front; RH: right hind; SED: single enzyme deficiencies; SMI31/ SMI32: Sternberger monoclonal incorporated antibody 31/ 32; VGaT: vesicular GABA transporter; VGluT: vesicular glutamate transporter; VLCFA: very long-chain fatty acids; WT: wild type. 


\section{INTRODUCTION}

Adequate cerebellar functioning depends largely on the Purkinje cells (PCs), the sole output neurons of the cerebellar cortex. PC dysfunction gives rise to 'cerebellar ataxia', a term generally used for a range of neurological disorders that affect balance, coordination and speech. Currently, more than 60 different types of cerebellar-based ataxias have been identified of which Friedreich's and spinocerebellar ataxias are the most common. Less well-known is that cerebellar defects are also prominent features in many peroxisomal disorders, a group of heterogeneous and rare diseases caused by dysfunction of peroxisomal proteins. Both in peroxisome biogenesis disorders (PBD) and in single enzyme deficiencies (SED), developmental or degenerative cerebellar pathologies manifest as part of a multi-organ disease (recently reviewed in De Munter et al. (De Munter et al., 2015).

More remarkable is that several patients who present with cerebellar ataxia and atrophy as the primary pathology were recently found to have mutations in the gene $17 \beta$-hydroxysteroid dehydrogenase type 4 (HSD17B4), encoding the central enzyme of the peroxisomal $\beta$-oxidation pathway, known as Dbifunctional protein or multifunctional protein-2 (further denoted as MFP2). MFP2 is indispensable for the breakdown of several substrates, including very long-chain fatty acids (VLCFA), branched-chain fatty acids, bile acid intermediates, and for both the degradation and synthesis of poly-unsaturated fatty acids (PUFAs). When the enzyme is completely inactive, this results in a severe neurodevelopmental disease characterized by hypotonia and brain malformations, frequently involving the cerebellum and brainstem (Ferdinandusse et al., 2006). Diagnosing the mildly affected MFP2 patients with ataxia was notoriously difficult as the metabolites that accumulate in plasma of the severe variants of the disease, such as VLCFA and branched-chain fatty acids, occurred in (near) normal levels. It is thanks to the application of next generation sequencing that the genetic defect could be defined (Lieber et al., 2014; Lines et al., 2014; McMillan et al., 2012). In the absence of relevant metabolite deregulation in plasma, the mechanisms underlying the cerebellar pathology are fully obscure.

Our previous studies have demonstrated that cerebellar pathology also manifests in $M f p 2^{-/-}$mice. We have demonstrated a mild delay in cerebellar foliation with impaired granule cell migration and an increased number of apoptotic cells in $M f p 2^{-/}$mice, indicating a role of peroxisomal $\beta$-oxidation in cerebellar development (Krysko et al., 2007). Moreover, starting at 4 weeks of age, they display poor 
rotarod performance and an uncoordinated locomotion with abnormal paw placement. Their motor phenotype is progressive and culminates in severe ataxia accompanied by PC degeneration and cerebellar atrophy (Verheijden et al., 2013).

In order to decipher the pathomechanisms of cerebellar degeneration in MFP2 deficiency, we recently generated Nestin-Mfp $2^{-/}$mice with neural selective MFP2 deletion. These mice also displayed coordination defects upon rotarod testing from the age of 4 weeks, proving that the cerebellar phenotype is not caused by peripheral MFP2 deficiency, as for example bile acid abnormalities (Verheijden et al., 2013). Here we investigated cerebellar structure and function in early symptomatic Nestin-Mfp $2^{-/}$mice. Through in-depth behavioral, histological and electrophysiological studies, we revealed a complex cerebellar phenotype.

\section{MATERIALS AND METHODS}

\section{Mouse breeding}

The generation of $M f p 2^{-/}$and Nestin-Mfp $2^{-/}$mice has been described elsewhere (Baes et al., 2000; Verheijden et al., 2013). Mice were bred in the animal housing facility of the KU Leuven, had ad libitum access to water and standard rodent food and were kept on a 12 hour light and dark cycle. Genotyping was performed on tail DNA. All animal experiments were performed in accordance with the "Guidelines for Care and Use of Experimental Animals" and fully approved by the Institutional Animal Ethical Committee of the KU Leuven (\#177/2012 and \#122/2015).

\section{Motor coordination assessment}

\section{Rotarod}

An accelerating rotarod (Med Associates) was used to test motor skill learning in 4-week-old Nestin$M f p 2^{-/}$mice and their respective controls. The rotarod test included a short adaptation session in which the mice were trained to stay on a steady rod for at least 30 s. Trials were performed on 5 consecutive days and included 4 tests with an acceleration of $4-40 \mathrm{rpm}$ in $300 \mathrm{~s}$. Mice were allowed to rest for 1 hour in between each trial. Falling of the rod or reaching 300s were considered as the trial ending. A cohort of 6 male mice per genotype was used. 


\section{Noldus CatWalk}

The CatWalk system (Noldus Information Technology) was used to assess locomotor gait dynamics in 4-week-old Nestin-Mfp $2^{-/-}$mice and wild type (WT) littermates as described previously (Vandeputte et al., 2010). Briefly, mice were given 3 trials in which they were allowed to cross the pressure-sensitive plate of the CatWalk system in an unforced manner. Uninterrupted tracks with at least 4 cycles of complete steps were considered successful. Speed variation was less than $25 \%$ between both genotypes. The animal's paw prints were given a label (right-fore (RF), right-hind (RH), left-fore (LF), left-hind (LH)) and subsequently analyzed using the CatWalk software. Stride length and base of support (BOS) were recorded and averaged over the 3 trials. A cohort of 4 male mice per genotype was used.

\section{Balance beam test}

The balance beam was used to assess fine motor coordination and the ability to maintain balance while traversing a narrow beam to a safe platform. The apparatus consisted of square beams with a flat surface and one meter in length, positioned $50 \mathrm{~cm}$ above the table top. Beams had a cross section of $30 \mathrm{~mm}$ or $5 \mathrm{~mm}$. Mice were trained 4 times a day for 3 consecutive days on the $30 \mathrm{~mm}$ beam until they were able to cross within $30 \mathrm{~s}$ without hesitation. On the testing day, the time needed to cross the $5 \mathrm{~mm}$ beam and the number of paw slips were recorded. A cohort of 6 male mice per genotype was used.

\section{Histology and Immunohistochemistry}

Animals were deeply anesthetized with a mix of Dormitor $(1 \mathrm{mg} / \mathrm{kg})$ and Nimatek $(75 \mathrm{mg} / \mathrm{kg})$ and perfused transcardially with phosphate buffered saline (PBS, $\mathrm{pH}$ 7.4), followed by $4 \%$ paraformaldehyde (PFA). Brains were isolated, post-fixed overnight in 4\% PFA and cryopreserved in $20 \%(\mathrm{wt} / \mathrm{vol})$ sucrose. Brains were embedded in a sagittal orientation in TissueTek (Thermo Scientific), rapidly frozen and stored at $-20^{\circ} \mathrm{C}$. Free floating sections $(40 \mu \mathrm{m})$ were cut on a cryostat and stored in cryoprotectant until further processing. Double stainings of calbindin with either vesicular glutamate transporter 1 (VGluT1), - VGluT2 or - vesicular gamma-aminobutyric acid (GABA) transporter (VGaT) were performed as described, with slight modifications (Ramer et al., 2010). Sections of the cerebellar vermis were blocked for 30 mins in 10\% normal goat serum (Vector Laboratories), $0.1 \%$ Triton X-100 
in PBS and incubated overnight at $4^{\circ} \mathrm{C}$ with the primary antibodies in block (mouse calbindin (Sigma; 1:200), rabbit VGLuT1 (Synaptic Systems; 1:1000), rabbit VGluT2 (Synaptic Systems; 1:1000) or rabbit VGaT (Synaptic Systems; 1:1000)). After extensive washing with PBS, sections were incubated with the appropriate secondary antibodies in block for 2 hours at room temperature (goat-anti-mouse Alexa-Fluor-568 (Invitrogen, 1:200) or goat-anti-rabbit Alexa-Fluor-488 (Invitrogen, 1:200)). Floating sections were transferred to Superfrost Plus (Thermo Scientific) slides and coverslipped with Vectashield mounting medium containing 4',6-diamidino-2-phenylindole (DAPI) (Vector Laboratories). Images were acquired with a Zeiss AxioCam camera connected to a Zeiss confocal laser scanning microscope 510 with a $63 x$ oil immersion objective. The pinhole was set at 1 arbitrary unit. Confocal image acquisition consisted of images in the z-direction with a step size of $2 \mu \mathrm{m}$.

Stainings on paraffin and cryosections were performed as described (Hulshagen et al., 2008; Verheijden et al., 2013) and imaged on a motorized inverted IX-81 microscope connected to a CCD-FV2T digital camera (Olympus). A cohort of 4 mice per genotype was used.

\section{Quantitative Measurements}

The external granule cell layer (EGL) and molecular layer (ML) thickness were determined using 20X images from haematoxylin and eosin (H\&E) stained cerebellar sections. The thickness of the ML was calculated as the distance between the base of the PC soma and the external border of the ML. EGL thickness was determined relative to the ML. Measurements were performed in duplicate on 10 pictures, 1 for each cerebellar lobule, resulting in 20 measurements per animal. VGluT1 density was measured within a fixed rectangle positioned in the distal part of the PC dendritic tree. The number of VGluT2 and VGaT immunoreactive boutons was analyzed in the ML and corrected for the area selected. Analyses were performed on 3 pictures per cerebellar lobule and on 3 consecutive images of the z-stack, resulting in 90 measurements per animal. Results were averaged per lobule, resulting in 40 measurements per genotype. Climbing fiber (CF) extension was measured from the base of the PC soma to the tip of the $\mathrm{CF}$ arbor relative to the ML thickness in 30 pictures per genotype. All measurements were performed using the FiJi software. A cohort of 4 mice per genotype was used. 


\section{Immunoblotting}

Western blotting experiments were conducted as described (Baes et al., 2000). A calbindin antibody (Sigma) was used at a concentration of $1 / 1000$. The $\beta$-actin antibody (Abcam) was used at a concentration of 1/5000. Secondary antibodies were horseradish peroxidase (HRP) labeled and detection was performed using the Enhanced Chemiluminescence (ECL) plus detection kit (Amersham).

\section{Electrophysiology}

The preparation of acute cerebellar slices was performed as previously described (Petrinovic et al., 2013), with slight modifications. Briefly, postnatal day (P) 21 - P28 mice were anesthetized with halothane, decapitated and cerebella were quickly dissected. Sagittal slices of the cerebellar vermis (200 $\mu \mathrm{m})$ were cut on a vibratome (Leica) and collected in ice-cold, oxygenated extracellular solution containing the following (mM): 140 Choline Chloride, $26 \mathrm{NaHCO}_{3}, 14$ glucose, $7 \mathrm{MgCl}_{2}, 2.5 \mathrm{KCl}, 1.25$ $\mathrm{NaH}_{2} \mathrm{PO}_{4} * \mathrm{H}_{2} \mathrm{O}$ and $0.5 \mathrm{CaCl}_{2}$. Slices were transferred to oxygenated extracellular solution and kept at $34^{\circ} \mathrm{C}$ for at least 30 mins before being transferred to the recording chamber which was continuously provided with fresh oxygenated extracellular solution at room temperature. PCs were visualized using an upright microscope (Zeiss; Axioskop 2). All recordings have been made using the whole cell configuration of the patch clamp technique. Patch pipettes were made of borosilicate glass (Hilgenberg) with resistances of 2-6 M $\Omega$ when filled with the patch pipette solution containing the following $(\mathrm{mM})$ : $120 \mathrm{NaCl}, 26 \mathrm{NaHCO}_{3}, 11$ glucose, $2 \mathrm{KCl}, 2 \mathrm{CaCl}_{2}, 1.19 \mathrm{MgSO}_{4} * 7 \mathrm{H}_{2} \mathrm{O}$ and $1.18 \mathrm{KH}_{2} \mathrm{PO}_{4}$. Recordings were performed for both current- and voltage-clamp modes using an EPC 10 amplifier (HEKA). Stimulus generation and data acquisition were made with PatchMaster software (HEKA). Spontaneous and depolarization-evoked potential signals were filtered at $4 \mathrm{kHz}$ and digitally sampled at $20 \mathrm{kHz}$. Spontaneous action potential firing frequency was analyzed from current clamp recordings with a $0 \mathrm{pA}$ injected current. Intrinsic excitability was investigated by setting membrane potential at $-80 \mathrm{mV}$ and injecting 1 second steps of depolarizing current (from 0 to $2.5 \mathrm{nA}$ in $50 \mathrm{pA}$ increment). When injected with a sufficient depolarizing current, PCs generated repetitive spikes. Action potential frequency was measured for each injected current intensity. The average frequency over the time of current injection was measured by dividing the number of interspike intervals by the time interval between the first and 
the last spike. These values were used to construct current-frequency plots. Passive cellular parameters were extracted from voltage clamp traces by analyzing current relaxation induced by a $10 \mathrm{mV}$ step change from a holding potential of $-70 \mathrm{mV}$. For the estimation of the cell capacitance, only the slow component of the capacitive currents, corresponding to the dendritic compartment, was taken into account as the fast somatic component only contributes to about $2 \%$ of the total capacitive charge (Llano et al., 1991). Recordings and data analysis were conducted as described on 8 WT and 9 Nestin-Mfp $2^{-/-}$ PCs.

\section{Biocytin labeling and PC morphological analysis}

Cerebellar sections used for electrophysiological recordings were simultaneously used for PC morphological reconstruction by means of Biocytin injection (0.5\%, Sigma-Aldrich). Slices were immersion fixed overnight in $4 \%$ fresh PFA and Biocytin was revealed by means of cytochemistry using a streptavidin-488 secondary antibody (Jackson Immunoresearch, 1/200 in PBS-T). Images were acquired using a Zeiss confocal laser scanning microscope 510 with a $63 \mathrm{x}$ oil immersion objective in the z-direction with a step size of $1 \mu \mathrm{m}$. Neuronstudio (version 9.92) was used to accurately reconstruct the PC dendritic tree and to determine PC Strahler orders as described previously (Chen et al., 2013), with slight modifications. The FiJi program was used for Sholl analysis by applying the Simple Neurite Tracer plugin (Chen et al., 2013). For each genotype, 5 PCs were reconstructed and analyzed.

\section{Golgi staining}

The Golgi impregnation method was applied with the Rapid Golgi stain kit (FD Neurotechnologies). Briefly, 4-week-old WT and Nestin-Mfp $2^{-/}$mice were anesthetized and cerebella were quickly dissected, cut in the sagittal plane and handled according to the manufacturer's protocol. Cerebellar halves were embedded in $4 \%$ agarose and $100 \mu \mathrm{m}$ sections were cut on a vibratome, stained and transferred on Superfrost Plus slides. Z-stack images were taken on a motorized inverted IX-81 microscope connected to a CCD-FV2T digital camera (Olympus). PC soma size was determined as described previously (Tavazoie et al., 2005). Spine density and length were averaged for 3 terminal dendrites for each PC. Spine number was counted and corrected for dendrite length. Spine length was 
averaged for 6 spines per dendrite and calculated as the distance between the base of de dendrite and the outmost distal point of the spine head. Analyses were performed on 15 PCs per genotype, from 3 WT and 3 Nestin-Mfp2 $2^{-/-}$mice, using the FiJi software.

\section{Electron microscopic analysis}

Animals were deeply anesthetized with a mix of Dormitor $(1 \mathrm{mg} / \mathrm{kg})$ and Nimatek $(75 \mathrm{mg} / \mathrm{kg})$ and perfused transcardially with PBS ( $\mathrm{pH} 7.4$ ), followed by a solution containing $2 \%$ PFA and $2 \%$ glutaraldehyde in $0.1 \mathrm{M}$ sodium cacodylate ( $\mathrm{pH}$ 7.4). Cerebella from $2 \mathrm{WT}$ and 2 Nestin-Mfp $2^{-/}$mice were isolated, sectioned sagitally and post-fixed overnight by immersion in $4 \%$ glutaraldehyde in $0.1 \mathrm{M}$ sodium cacodylate buffer ( $\mathrm{pH}$ 7.4) for 1 day and then at $4{ }^{\circ} \mathrm{C}$ for 2 days. Following previously described methods and protocols (da Silva et al., 2014), samples were processed for light and electron microscopy. Semi-thin sections of cerebella were stained with p-phenylenediamine (PPD) to allow the visualization of axon swellings in myelinated axons, and determination of regions to be analyzed by electron microscopy. Ultrathin sections (approximately $60 \mathrm{~nm}$ thick) encompassing the granular, PC and molecular layers of lobe $\mathrm{V}$ at the cerebellar vermis were used in all ultrastructural analyses. The degree of myelination was determined by analyzing the g-ratio in the cerebellar white matter of WT $(n=297$ axons) and Nestin-Mfp2 $2^{-/-}$( $=232$ axons) mice as described (da Silva et al., 2014).

\section{Statistical analysis}

All data were analyzed with GraphPad Prism software (version 5.0). Statistical analyses were performed using the unpaired, two-sided Student's $t$-test or with one-way ANOVA repeated measures or two-way ANOVA repeated measures followed by the Bonferroni post hoc test. Data are shown as the mean \pm SEM and statistical significance was set at $\mathrm{P}<0,05$.

\section{RESULTS}

\section{Nestin-Mfp $2^{-/}$mice show cerebellar ataxia and mild disturbances in motor learning}

We previously reported that Nestin-Mfp $2^{-/-}$mice underperform on the rotarod at the age of 4 weeks (Verheijden et al., 2013). To distinguish whether this is due to the inability to learn complex motor skills 
(Bergeron et al., 2014; Bureau et al., 2010) or due to pure cerebellar ataxia, 4-week-old mice were trained 4 times per day on an accelerating rotarod during 5 consecutive days. WT mice rapidly enhanced their performance, reaching the maximal latency of 5 minutes during the final trial of the first training day (Supplementary Figure 1), which remained constant in all subsequent tests. In contrast, Nestin-Mfp2- mice showed a slight delay in learning the new motor task as they still improved their scores during the second and third training days (Figure 1A). Interestingly, there was a clear tendency that Nestin$M f p 2^{-/}$mice performed worse in the last as compared to the first test of the day (Supplementary Figure $1)$.

To gain more evidence for ataxic behavior we performed CatWalk analysis, an automated gait analysis system, and used a balancing beam test which is more sensitive in revealing subtle coordination defects related to cerebellar dysfunction (Brooks and Dunnett, 2009; Herson et al., 2003). Gait analysis revealed significant decreases in stride length and base of support (BOS) of the hind paws in Nestin-Mfp $2^{-/}$mice (Figure 1B, C). Moreover, Nestin-Mfp $2^{-/}$mice made more slips and needed more time to cross the balancing beam as compared to controls (Figure 1D, E). The mild impairment in motor learning of Nestin-Mfp $2^{-/}$mice and their coordination problems on the CatWalk, rotarod and balance beam are suggestive of a cerebellar defect at this young age.

\section{Cerebellar development and gross architecture are unaltered in Nestin-Mfp $2^{-/-}$mice}

The early-onset coordination problems of Nestin-Mfp $2^{-/-}$mice were suggestive of impaired cerebellar development, which occurs postnatally over a three-week period in mice. Deficits in cerebellar maturation are often accompanied with a delay in the formation of the characteristic folia and impairment in granule cell migration. During its development, the cerebellar cortex consists of an extra outermost layer called the EGL, which gradually becomes thinner as the granule cells migrate along the Bergman glia fibers to form the internal granule cell layer (IGL).

We compared the gross morphology of WT and Nestin-Mfp $2^{-/}$cerebella on sagittal sections at different postnatal ages. At P8, a mature foliation pattern with well-defined fissures of similar depth separating the characteristic folia was observed in WT and Nestin-Mfp $2^{-/}$cerebella (Figure 2A). Furthermore, the thickness of the IGL and the ratio EGL/ ML were unaltered in Nestin-Mfp $2^{-/-}$mice, indicating normal 
granule cell migration (Figure 2C, G, H). PC outgrowth was examined after calbindin staining. This did not reveal obvious abnormalities in PC alignment and morphology with the majority of the cells presenting with a primary dendrite branching to a similar extent in the parasagittal plane (Figure 2E). Also, at 4 weeks of age, cerebellar weight and size (data not shown) were comparable between Nestin$M f p 2^{-/}$and WT mice with a normal appearance of the cortical layers and an equal thickness of the ML (Figure 2B, D, F, I). Calbindin immunoreactivity by histological staining and western blot was unaltered in Nestin-Mfp $2^{-/}$mice, demonstrating the absence of generalized PC loss at an age of 4 weeks (Figure 2F, J, K). These results indicate that the early-onset motor impairment in Nestin-Mfp $2^{-/}$mice cannot be associated with gross alterations in cerebellar morphology.

\section{Axonal transport is compromised in 4-week-old Nestin-Mfp $2^{-/-}$mice}

Interestingly, calbindin staining showed numerous focal spheroids present on PC axons of 4-week-old Nestin-Mfp $2^{-/}$mice, some of them being 5 to 10 times wider compared to the uniform PC axons in WT littermates (Figure 3C, D). These axonal abnormalities were also observed, although to a lesser extent in 2-week-old Nestin-Mfp2 $2^{-/}$cerebella (Figure 3A, B), indicating progressive alterations of axon structure in Nestin-Mfp $2^{-/-}$mice. On semi-thin sections, axonal swellings were clearly detected on myelinated axons of Nestin-Mfp $2^{-/-}$PC (Figure 4A). Myelination assessment by determination of the gratio revealed normal myelin levels in cerebellar white matter of Nestin-Mfp $2^{-/-}$mice (Figure 4B). Ultrastructural analysis revealed the presence of normal-appearing PC axons in Nestin-Mfp $2^{-/-}$mice (Figure 4C). However, numerous axonal swellings could be easily visualized in the granular layer of Nestin-Mfp2 $2^{-/-}$mice (Figure 4D). The swellings were 2 - 3 times the width of axons, and were characterized by the accumulation of subcellular organelles. The accumulation of mitochondria and membrane stacks was prominent (Figure 4D), and are suggestive of impaired axonal transport in PC axons of Nestin-Mfp $2^{-/-}$mice.

These findings prompted us to examine the axonal status in other white matter tracts. Staining of a series of coronal sections through the corpus callosum of 4-weeks-old Nestin-Mfp $2^{-/}$and WT littermates with the Sternberger monoclonal incorporated antibody 31 (SMI31) showed a normal neurofilament pattern in healthy looking axons in both genotypes. In agreement, SMI32 staining, a marker for non- 
phosphorylated neurofilament and indicative of axonal damage, was undetectable. Furthermore, the absence of anti-Alzheimer precursor protein (APP) accumulation in axons of 4-weeks-old Nestin-Mfp2${ }^{-}$mice proved that axonal transport is uninterrupted in the corpus callosum (Supplementary Figure 2). Based on these findings, we conclude that axonal abnormalities in juvenile Nestin-Mfp $2^{-/}$mice are restricted to cerebellar PCs.

\section{PC firing frequency and excitability is reduced in early-symptomatic Nestin-Mfp $2^{-/-}$mice}

An ataxic phenotype at a young age can be caused by physiological dysfunction of cerebellar PCs (Hourez et al., 2011; Milnerwood et al., 2010). We aimed at investigating whether the uncoordinated phenotype in young Nestin-Mfp $2^{-/}$mice correlated with PC electrophysiological dysfunction. Therefore, the functional properties of 3 to 4 -week-old WT and Nestin-Mfp $2^{-/}$PCs were studied in slices of the cerebellar vermis.

Supplementary Table 1 shows the parameters from the patch clamp recordings. MFP2 deletion did not alter the membrane resistance $(\mathrm{GOhm})$ nor the resting membrane potential $(\mathrm{mV})$. However, membrane capacitance (pF) of MFP2 deficient PCs was significantly increased (Figure 5A).

In the absence of excitatory synaptic inputs, healthy PCs exhibit a spontaneous firing pattern characterized by the presence of rapid and highly regular action potentials. Whole cell configuration recordings showed a significant reduction in PC spontaneous activity in the absence of MFP2 (Figure 5B). To further test PC excitability, current-frequency plots were made. For this, membrane potential was initially held at $-80 \mathrm{mV}$ and stepwise depolarized with $50 \mathrm{pA}$ injections. Our data showed a significant decrease in Nestin-Mfp $2^{-\digamma}$ PC excitability with less action potentials generated for each current injected (Figure 5C). To summarize, we show that impaired peroxisomal $\beta$-oxidation results in early-onset PC dysfunction with significant decreases in firing frequency and intrinsic excitability and an increased membrane capacitance.

\section{Subtle alterations in PC morphology in 4-week-old Nestin-Mfp $2^{-/-}$mice}

Because the membrane capacitance is primarily determined by the cell surface area, we checked whether the observed increase in capacitance in MFP2 deficient PCs was related to alterations in PC morphology. 
After visualization of the biocytin injected in PCs during intracellular recordings (Figure 6A, D), neuronal morphology was accurately reconstructed in NeuronStudio and the density of branching points at different distances from the cell PC soma was assessed by means of Sholl analysis. As already suggested by the equal thickness of the ML in 4-week-old Nestin-Mfp2 $2^{-/}$mice (Figure 2I), the complexity of the PC dendritic tree was unaffected by impaired peroxisomal $\beta$-oxidation (Figure 6C). In agreement, Strahler orders of the dendritic branches were equal in WT and Nestin-Mfp $2^{-/-}$mice (Figure 6B, E, F).

The Golgi impregnation method was used to study PC dendritic spines and soma size (Figure 7A, B). While no difference was observed in the PC soma size (Figure 7E), Golgi staining revealed significant increases in spine length and density in 4-week-old Nestin-Mfp $2^{-/-}$mice (Figures 7C, D). The increased dendritic spine length of Nestin-Mfp $2^{-/} \mathrm{PC}$ was confirmed by ultrastructural analysis. (Figure 7F $-\mathrm{H}$ ). Together, our results indicate that the general morphology of the Nestin-Mfp $2^{-/}$PC soma and dendritic trees appears unaffected by the absence of MFP2 but changes are present at the level of the spines.

\section{MFP2 is indispensable for adequate cerebellar wiring}

Proper cerebellar functioning also depends largely on adequate wiring of the PCs. CF and mossy fibers (MF), originating from the inferior olivary nucleus and the spinal cord respectively, are the main afferents innervating these cells. During cerebellar development, CF innervation switches from a state of multiple innervation to PC monoinnervation through the elimination of supernumerary CFs, thereby making direct contacts to the proximal dendritic part of a single PC. In contrast, MF innervation occurs indirectly by making synaptic contacts onto granule cells, which in turn send parallel fibers (PF) contacting the distal part of the dendritic tree of multiple PCs. To examine whether the defective PC morphology is accompanied by a difference in synaptogenesis, immunohistochemical analysis was performed to visualize PC excitatory and inhibitory input.

Immunofluorescence double staining using calbindin and VGluT1 or VGluT2, marking PF and CF terminals respectively, was used in order to study differences in PC glutamatergic input (Figure 8A, B, D, E). In the cerebellar ML, both VGluT1 and VGluT2 immunoreactivities were significantly decreased in 4-week-old Nestin-Mfp2-/ mice (Figure 8G, J). Moreover, VGluT2 positive CF terminals were 
observed in a restricted area of the ML and on PC somata, indicating inadequate maturation of PC innervation (Figure 8H, I). Inhibitory synaptic contacts on the PC dendritic tree were unaffected, as shown by the equal number of VGaT in the cerebellar ML (Figure 8C, F, K).

\section{DISCUSSION}

We here show that intact peroxisomal $\beta$-oxidation is crucial for PC function in the juvenile mouse cerebellum. We find that mice lacking the key $\beta$-oxidation enzyme, MFP2, exhibit impaired motor learning and ataxia. This was associated with altered electrophysiological properties of PCs, PC axonal degeneration, and $\mathrm{CF}$ and PF wiring abnormalities in the absence of overt morphological alterations. Our data provide new functional insights with regard to the importance of peroxisomes in the central nervous system.

It is well established that full ablation of peroxisomal function both in man and in mice, through $P E X$ gene mutations, causes dysgenesis of the cerebellum with granule cell and PC migration defects and severe PC malformation (Barkovich and Peck, 1997; Faust, 2003; Muller et al., 2011). In addition, in recent years, adolescent and adult patients with mild PBD were identified in which cerebellar ataxia is a primary and recurrent feature (Gootjes et al., 2004; Mignarri et al., 2012; Raas-Rothschild et al., 2002; Regal et al., 2010; Sevin et al., 2011). The fact that several patients with a similar pathology were found to have mutations in the peroxisomal $\beta$-oxidation gene HSD17B4 (Lieber et al., 2014; Lines et al., 2014; McMillan et al., 2012; Pierce et al., 2010) indicated that this pathway is essential to maintain cerebellar integrity. However, given that no important metabolic changes were detected in the plasma of these patients, it remains fully obscure how a defect in peroxisomal $\beta$-oxidation can cause cerebellar degeneration. In order to obtain mechanistic insights, we phenotyped mice with neural selective deletion of MFP2, circumventing influences of peripheral enzyme deficiency. It must be underlined that the Nestin promotor driving Cre expression is also active in other progenitor cells besides those giving rise to neurons, oligodendrocytes and astrocytes (Harno et al., 2013). Nevertheless, it is interesting to note that, in contrast to constitutive $M f p 2^{-/-}$mice, gross cerebellar development including foliation, granule cell migration and PC maturation is unaffected in Nestin-Mfp $2^{-/-}$mice. The difference may stem from normal MFP2 function in the liver, where it is essential for several chain shortening processes of 
carboxylates including the formation of mature bile acids. This is in agreement with studies on Pex2-mice in which improvement of the bile acid profile by bile acid feeding partially corrected the cerebellar developmental defects (Faust et al., 2005).

PC axonal swellings are the earliest pathological events detected in Nestin-Mfp $2^{-/}$mice, occurring as early as 2 weeks after birth and progressively increasing in number thereafter. Such axon dilations are a typical sign of axonal dystrophy. Of interest, PC axons seem extremely vulnerable to metabolic changes as spheroids were absent from the corpus callosum of Nestin-Mfp $2^{-/}$mice of the same age, although it cannot be excluded that they arise there at a later age. This could be related to the relative abundance of MFP2 in PCs as compared to other brain regions shown in men, mice and porcine (Itoh et al., 2000; Itoh et al., 1999; Moller et al., 1999) but also to the fact that PC seem to be more vulnerable to metabolic dysfunction as also shown for lysosomal diseases.

As in other axonal degeneration pathologies, the PC spheroids contained mitochondria and endoplasmic reticulum membrane stacks consistent with a block in axonal transport. It is however unclear how peroxisomal $\beta$-oxidation dysfunction causes axonal transport defects. As myelination of Nestin-Mfp $2^{-/-}$ PC axons appeared normal, it is unlikely that the swellings were related to myelin anomalies as seen in several other examples of axonal demise (Redondo et al., 2015; Teigler et al., 2009). This is also in accordance with our previous findings that PC axons were not affected in oligodendrocyte selective $M f p 2^{-/}$mice (Verheijden et al., 2013). Axonal spheroids on PC were also reported in several murine models with lysosomal or autophagy dysfunction (Ko et al., 2005; Komatsu et al., 2007; Wang et al., 2006). As the swellings did not contain undigested lysosomal debris, the origin of these axonal lesions remained obscure. In these models the axonal dystrophy correlated with disease progression. Moreover, the axonal dilations clearly preceded and were believed to cause PC degeneration (Walkley et al., 2010). It is therefore possible that the PC degeneration and loss that occurs from the age of 6 months in Nestin$M f p 2^{-/}$mice (Verheijden et al., 2013) (and unpublished observations) is a consequence of axonal transport dysfunction from an early age. An impaired energy state is an alternate cause of axonal demise. Axonal transport is fueled both by glycolysis and by mitochondrial ATP production (Mahad et al., 2008; Mahad et al., 2009; Zala et al., 2013). Multiple links between primary peroxisomal dysfunction causing secondary mitochondrial deficits have been uncovered (Baarine et al., 2015; Dirkx et al., 2005; Kruska 
et al., 2015), but according to our EM observations the mitochondria in axonal spheroids of Nestin$M f p 2^{-/} \mathrm{PC}$ showed a normal structure. It can however not be excluded that more subtle functional mitochondrial impairments occur in MFP2 deficient PC axons.

The altered electrophysiological properties of Nestin- $\mathrm{Mp}^{-/} \mathrm{PCs}$ are other intriguing observations. The most likely explanation for the clear-cut increase in membrane capacitance is an expansion of the cell surface (Petrinovic et al., 2013). Whereas the size of the PC soma and dendritic arborization were not altered, an increase in spine length and density was observed, possibly accounting for the increased capacitance. As an additional explanation, we hypothesize that impaired peroxisomal $\beta$-oxidation might affect the membrane capacitance through altering the lipid or protein composition of the membrane (Gentet et al., 2000). At present, the accumulation of VLCFA is the only known metabolic change in the brain of patients and mice with peroxisomal $\beta$-oxidation defects. According to previous data generated in our lab there is, however, no good correlation between the accumulation of VLCFA and an ataxic phenotype. In a mouse model with oligodendrocyte selective inactivation of MFP2, levels of C26:0 were indeed increased whereas cerebellar functioning was normal (Verheijden et al., 2013). Accumulating branched-chain fatty acids that require peroxisomal $\beta$-oxidation for their degradation are also suspected to affect cerebellar function (Ferdinandusse et al., 2008). However, the regular mouse chow contains only minute amounts of these fatty acids or their precursors making it unlikely that they are causative.

The increase in membrane capacitance likely contributes to the reduced firing rate and excitability of the MFP2 deficient PCs, although we cannot exclude that additional mechanisms might play a role. Detailed analysis of the action potentials of Nestin-Mfp $2^{-/}$PCs could not reveal alterations in action potential amplitude and shape, suggesting that $\mathrm{Na}^{+}, \mathrm{K}^{+}$or $\mathrm{Ca}^{++}$channels operate properly. Certainly, this is the first demonstration that inactive peroxisomal $\beta$-oxidation affects the electrical activity of a neuron. Impaired wiring of CF and PF on PCs is a third consequence of MFP2 deficiency. The delayed relocation of CF synapses from the PC soma to the dendrites could originate from an impaired PF - CF interaction (Hashimoto and Kano, 2005), but could as well be due to the reduced PC electrical activity. Indeed, decreasing PC excitability by specifically expressing a chloride channel in PCs impaired the redistribution of CF synapses from PC somata to the proximal dendrites (Lorenzetto et al., 2009). We 
assume that the subtle changes in spine density and length at distal PC dendrites are a consequence of this altered interaction of PCs with their stimulatory input as was previously shown in a glutamate receptor $\delta 2$ subunit (GluR $\delta 2)$ knockout mouse model (Kurihara et al., 1997).

The behavioral analysis showed cerebellar ataxia with a delay in motor learning in 4-week-old Nestin$M f p 2^{-/}$mice. Except for the CF input to the cerebellar cortex, adequate PC functioning is a strong determinant in acquiring new motor skills (Nguyen-Vu et al., 2013). Based on our findings, we hypothesize a dual contribution of the impaired PC activity and an immature CF innervation pattern in causing the delay in motor learning. Although strongly indicative of pure cerebellar malfunctioning, motor learning and coordination deficits can also involve other brain areas and the peripheral nervous system. Closer investigations of these regions are highly desirable.

In summary, by demonstrating that loss of the peroxisomal $\beta$-oxidation enzyme MFP2 impairs PC function and axonal integrity in a normally structured cerebellum, we uncovered novel aspects of the importance of peroxisomes for central nervous system functioning. It will be important to determine whether MFP2 has a cell autonomous function in PCs and which metabolic factors are involved. Our findings also raise the question whether other neurons than PC are affected by MFP2 deficiency in a similar way. Further elucidation of underlying mechanisms in this mouse model should shed light on the cerebellar pathologies in an increasing number of patients with mild peroxisomal disorders that present with near normal metabolite levels. 


\section{FIGURES}

Figure 1

$\mathbf{A}$

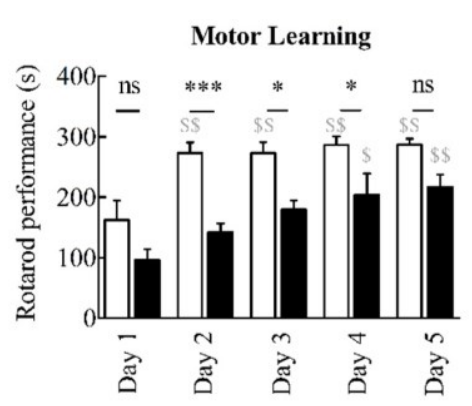

B

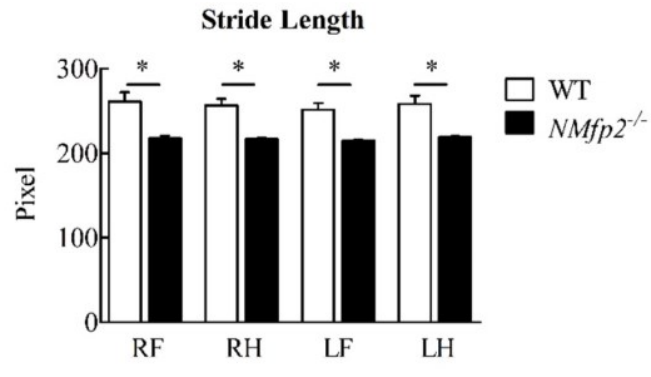

C

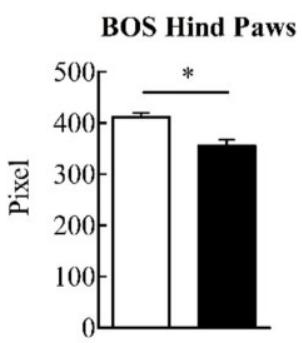

D

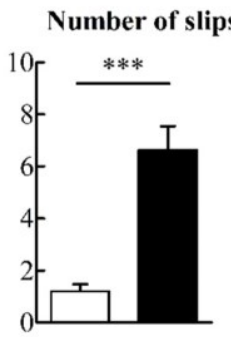

E Time to cross

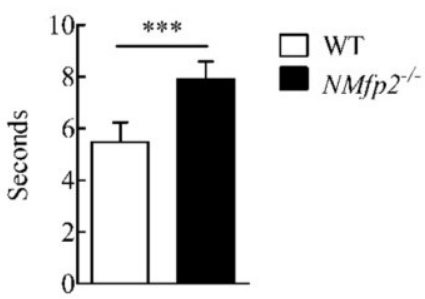

Figure 1: Altered motor behavior in 4-week-old Nestin-Mfp $2^{-/}$mice. (A) The performance during the first trial on the accelerating rod on 5 consecutive days is shown. Nestin-Mfp $2^{-/-}$mice are slower in acquiring motor skills as they improve until day 4. CatWalk analysis of stride length (B) and BOS (C) and balance beam analyses (D, E) confirmed cerebellar ataxia. $\mathrm{N}=6$ mice per group. Results are displayed as mean \pm SEM. * Compared to Nestin-Mfp $2^{-/}$on the same day, $\$$ compared to the first trial of the same genotype on the first day. NS: not significant; ${ }^{*} \mathrm{p}<0.05 ;{ }^{* * *} \mathrm{p}<0.001, \$ \mathrm{p}<0.05, \$ \$ \mathrm{p}<0.001$. 
Figure 2

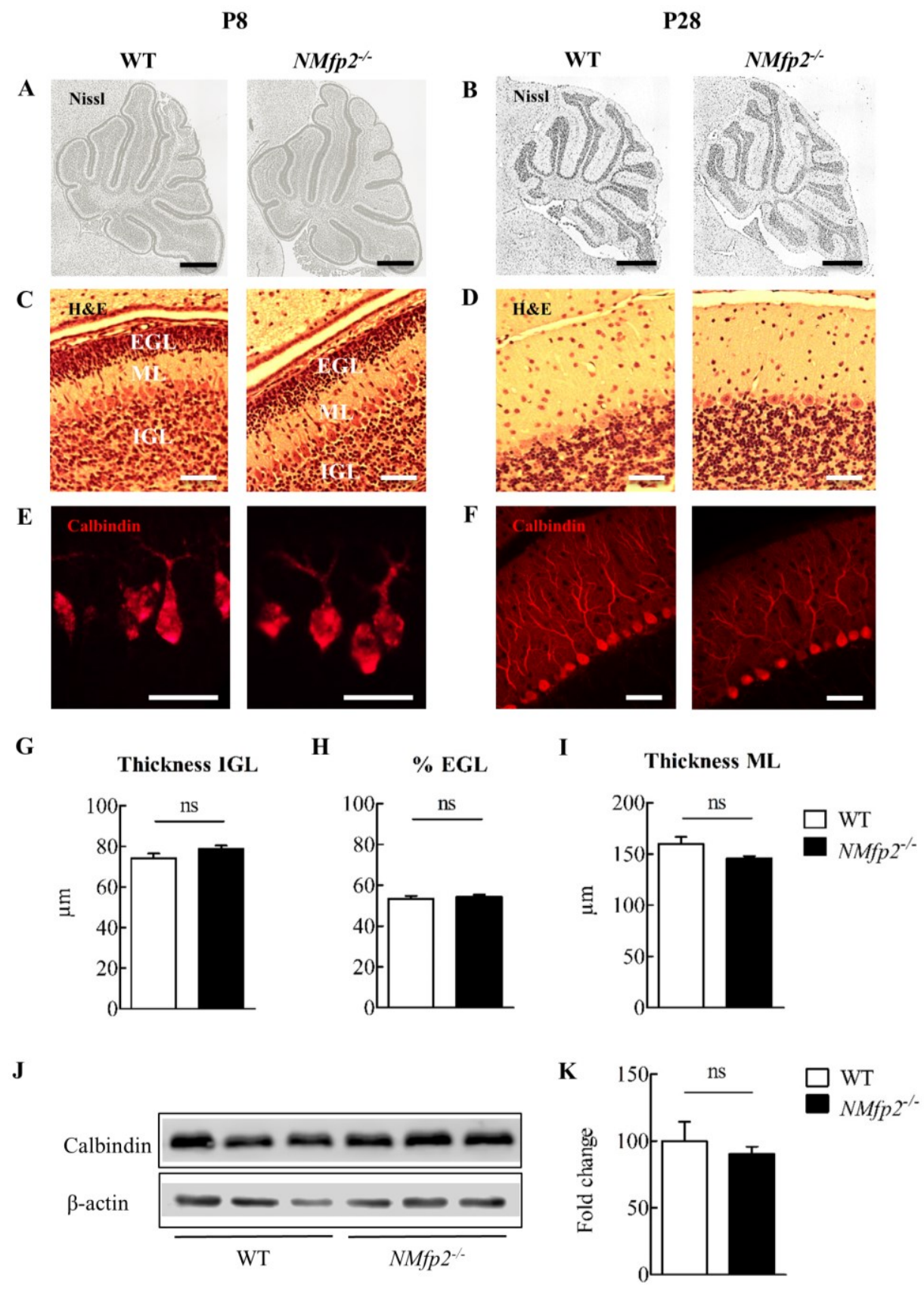

Figure 2: The gross morphology of the cerebellum is intact in the Nestin-Mfp $2^{-/-}$cerebellum. The cerebellar foliation pattern (A, B), thickness of cortical layers (C, D, G-I), and gross PC morphology (E, F) are similar in P8 and P28 WT and Nestin-Mfp $2^{-/}$mice. (J, K) Western blot analysis for calbindin shows equal levels in 4-week-old WT and Nestin-Mfp $2^{-/}$cerebella. $\mathrm{N}=4$ mice per group. Results are displayed as mean \pm SEM. NS: not significant. Scale bars: A: $500 \mu \mathrm{m}$; B: $1 \mathrm{~mm}$; C, D, E, F: $50 \mu \mathrm{m}$. 
Figure 3
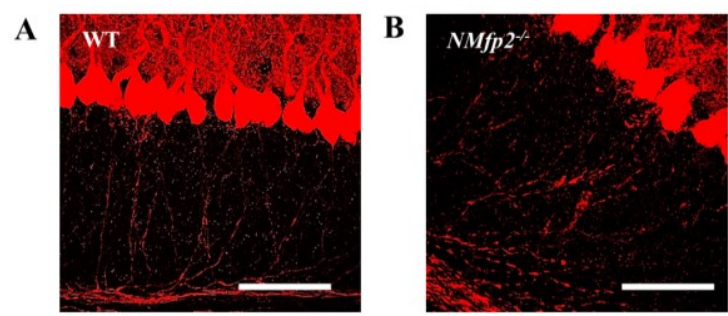

C

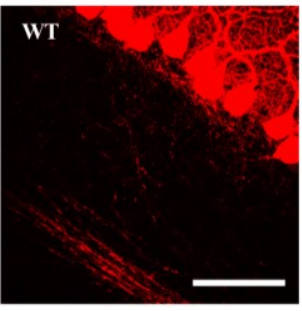

D

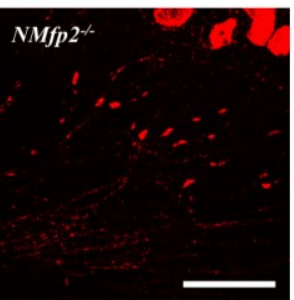

Figure 3: Abnormal PC axons in Nestin-Mfp $2^{-/-}$mice. (A-D) Calbindin staining revealed the presence of axonal swellings on PCs from Nestin-Mfp $2^{-/}$mice (B, D), which were more numerous at the age of 4 weeks (D) as compared to 2 weeks (B). $N=4$ mice per group. Scale bars: $50 \mu \mathrm{m}$. 
Figure 4
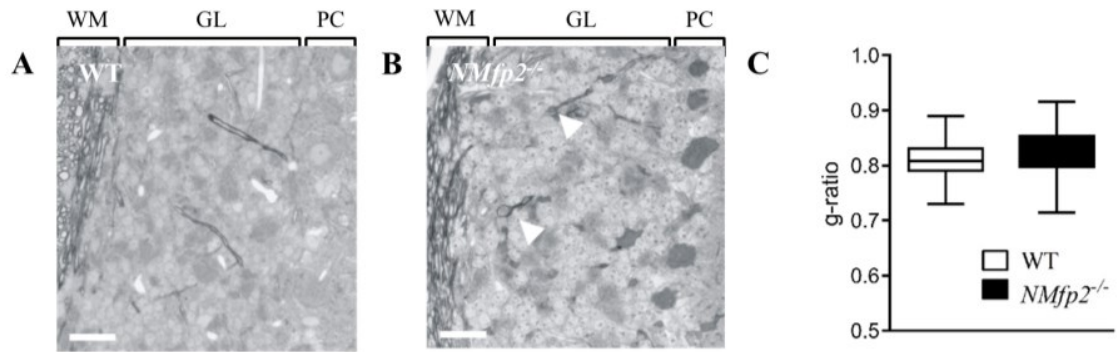

D

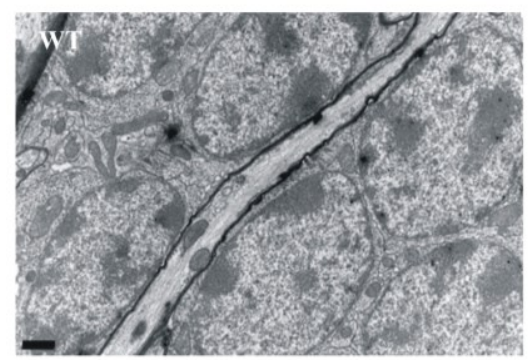

F

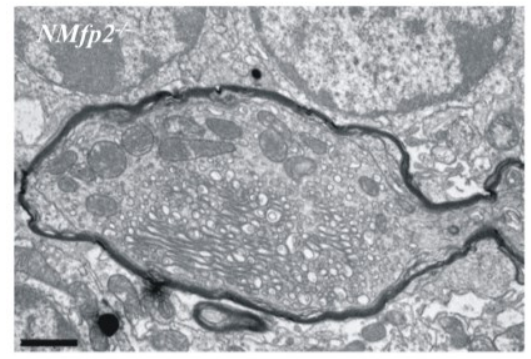

H

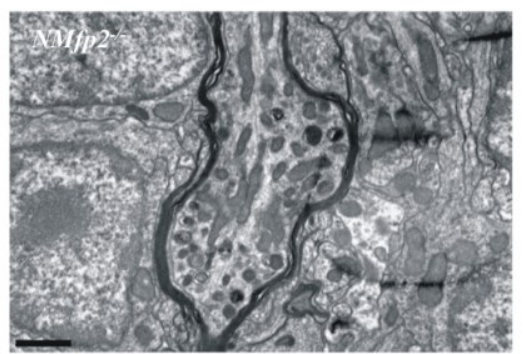

E

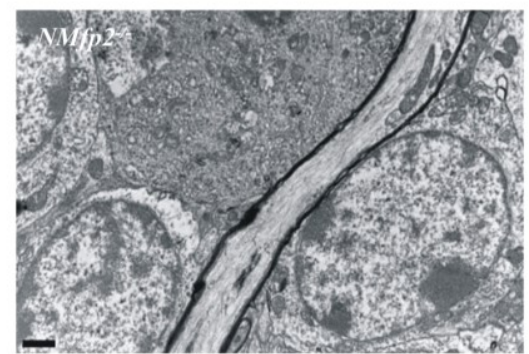

G

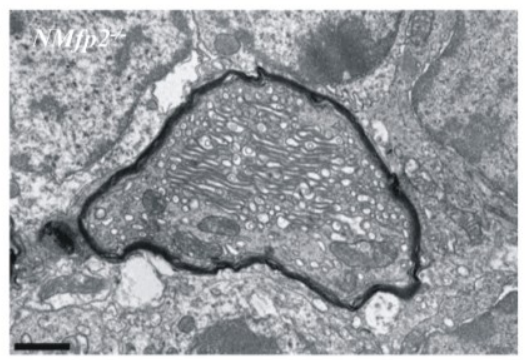

I

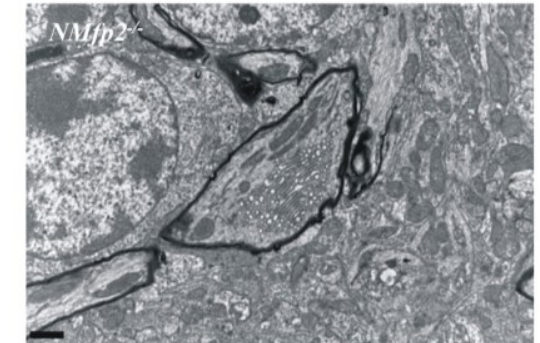

Figure 4: Axonal structure and organelle transport are compromised in Nestin-Mfp $2^{-/-}$mice. (A,

B) Semi-thin cerebellar sections from WT and Nestin-Mfp $2^{-/-}$mice stained with PPD revealed the presence of axonal swellings (white arrowheads) in myelinated PC axons of Nestin-Mfp $2^{-/ /}$mice. WM white matter; GL - granular layer; PC - Purkinje cell layer. (C) Quantification of myelination by g-ratio determination in cerebellar WM. (D, E) Ultrastructure of normal appearing myelinated PC axons crossing the granular layer of WT and Nestin-Mfp $2^{-/-}$mice. (F - I) Ultrastructure of axonal swellings in Nestin-Mfp $2^{-/-}$mice, containing an accumulation of several organelles, including mitochondria and membranous stacks, which are thought to accumulate due to defects in axonal transport. Scale bar A, B: $100 \mu \mathrm{m} ; \mathrm{D}-\mathrm{I}: 1 \mu \mathrm{m}$. 


\section{Figure 5}

A

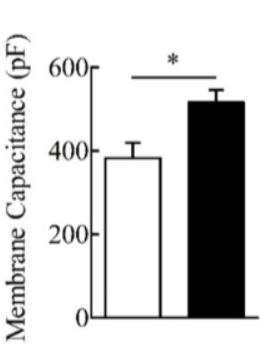

B

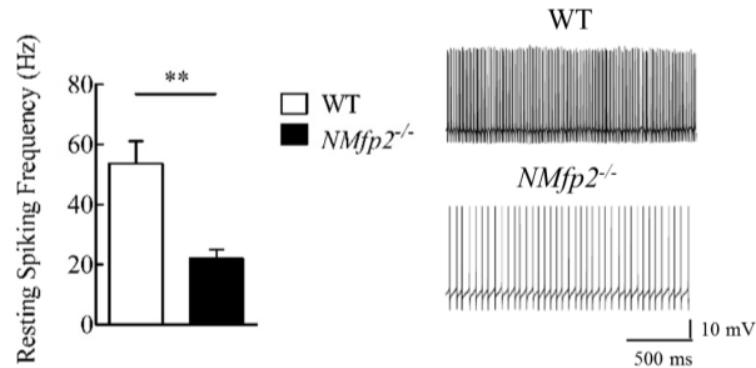

WT

C

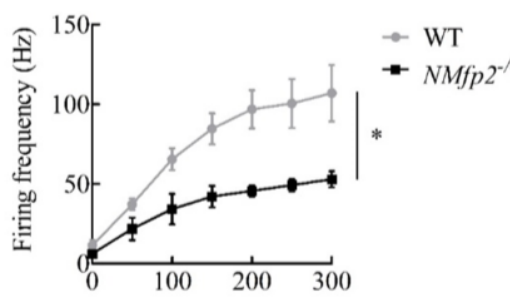

Injected current from rheobase $(\mathrm{pA})$

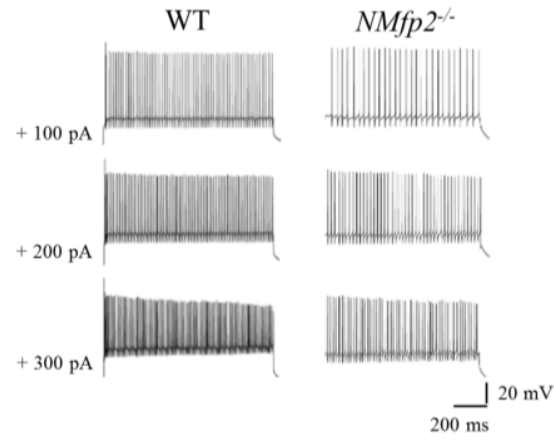

Figure 5: $M f p 2$ deletion alters $\mathrm{PC}$ electrophysiological properties. (A) Increased membrane capacitance in $M f p 2^{-/}$PCs. (B) PC resting spiking frequency is significantly reduced in 4-week-old Nestin-Mfp $2^{-/}$mice. (C) Current-frequency plots and corresponding traces demonstrate a significant decrease in PC intrinsic neuronal excitability. $\mathrm{N}=8$ - 9 cells per group. Results are displayed as mean \pm SEM. ${ }^{*} \mathrm{p}<0.05, * * \mathrm{p}<0.01$. 


\section{Figure 6}

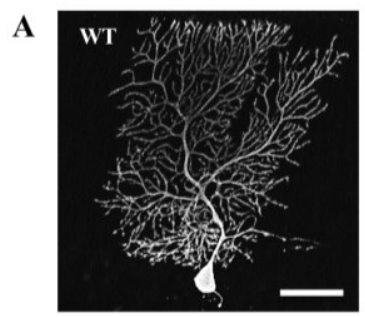

D

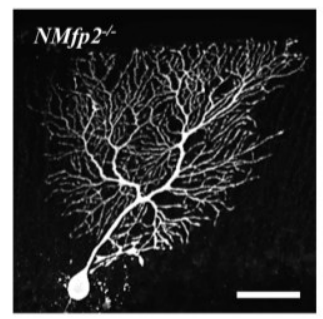

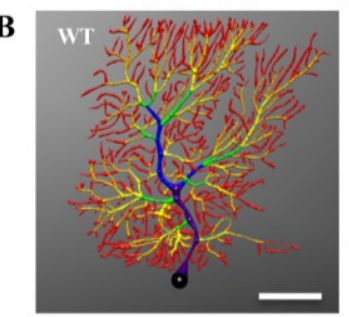

E

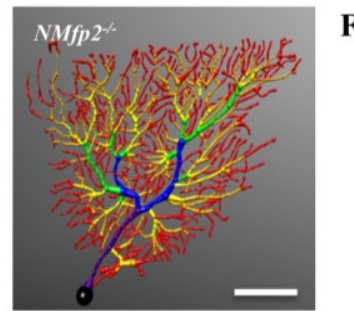

C

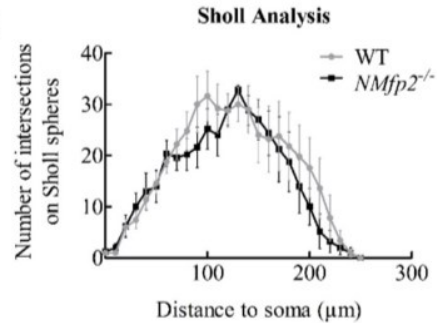

F

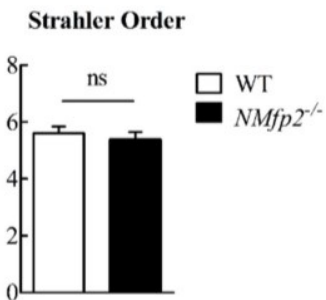

Figure 6: Detailed morphology of Nestin-Mfp $2^{--}$and WT PCs. (A, D) Visualization of PCs after immunological detection of injected biocytin. (B, E) Reconstructions of control and Nestin-Mfp $2^{-/-}$ dendritic trees allowing the analysis of Strahler orders, represented by the color codes. Depending on the branching complexity, branches closest to the cell soma are colored purple while PC terminal branches are depicted in red. Purple: order 1; blue: order 2; green: order 3; yellow: order 4; orange: order 5 and red: order 6. (C, F) Sholl and Strahler order analyses showed no differences in the complexity of the PC dendritic tree in the Nestin-Mfp $2^{-/-}$mice. $\mathrm{N}=5$ cells per group. Results are displayed as mean \pm SEM. NS: not significant. Scale bars: $50 \mu \mathrm{m}$. 


\section{Figure 7}

A

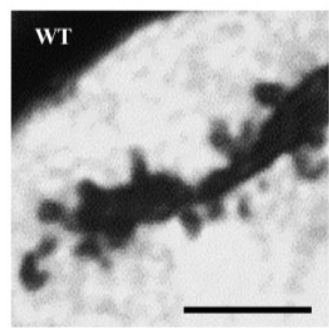

C

$$
\text { Spine Length }
$$

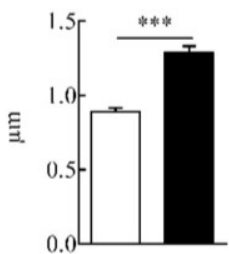

F

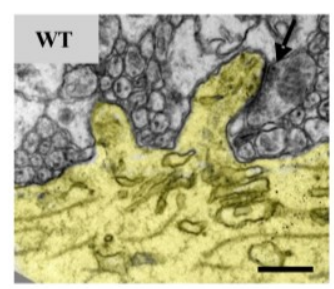

B

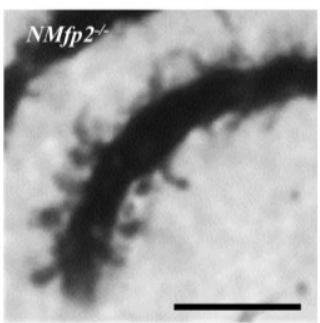

D
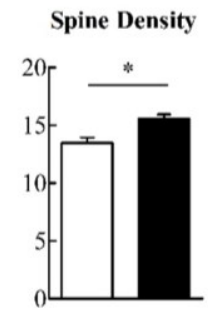

G

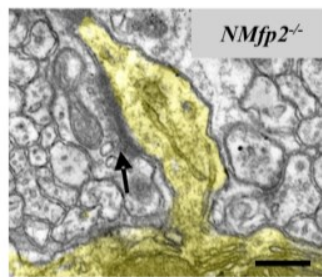

$\mathbf{E}$

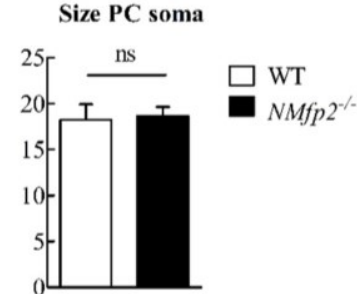

$\mathbf{H}$

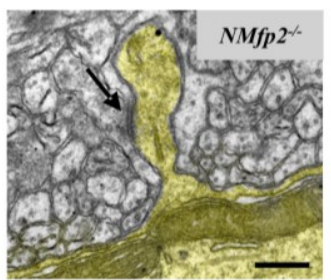

Figure 7: Subtle morphological alterations in PCs of young Nestin-Mfp $2^{-/}$mice. (A - E) Golgi staining revealed alterations in spine morphology and density but not in the size of the PC soma in the absence of MFP2. $\mathrm{N}=15$ cells per group. $(\mathrm{F}-\mathrm{H})$ Ultrastructural analysis of $\mathrm{PC}$ dendritic spines from WT and Nestin-Mfp $2^{-/}$mice. Dendrites were pseudo-colored yellow and arrows point to the active zones and postsynaptic densities. Results are displayed as mean \pm SEM. NS: not significant; ${ }^{*} p<0.05$; ***p $<0.001$. Scale bars A - B: $5 \mu \mathrm{m}, \mathrm{F}-\mathrm{H}: 0,4 \mu \mathrm{m}$. 
Figure 8

A

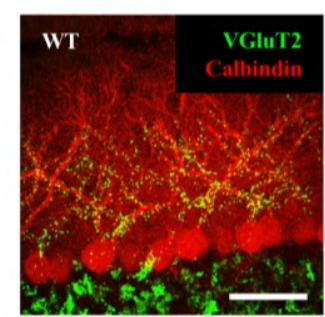

D

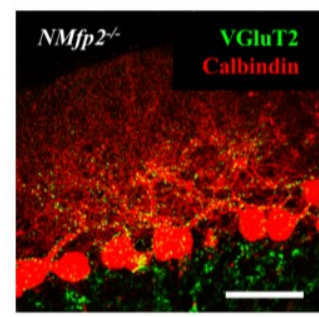

G

CF synapses/ area

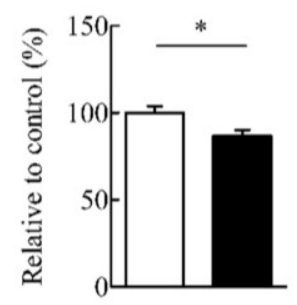

$\mathbf{J}$

PF synapses/ area

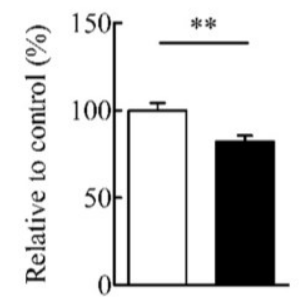

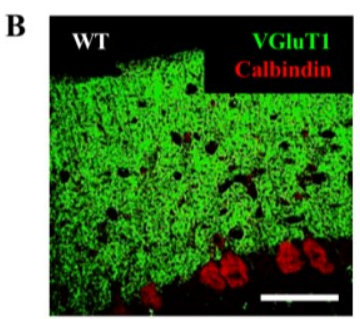

E

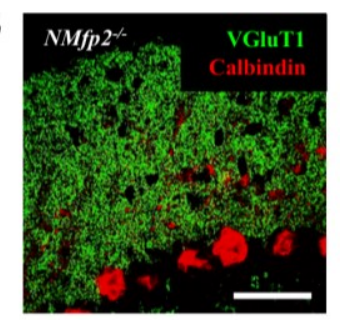

H

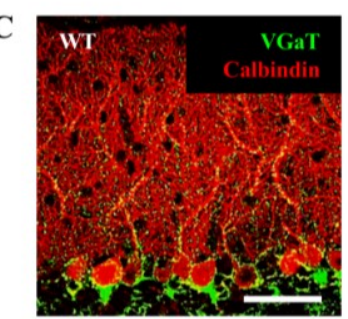

F

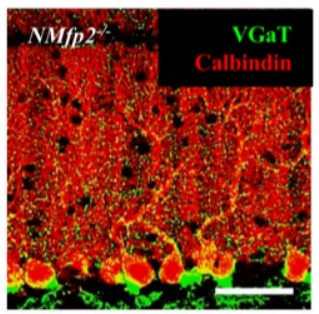

CF synapses/ PC soma

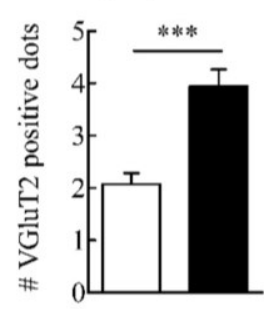

K GABAergic synapses/ area

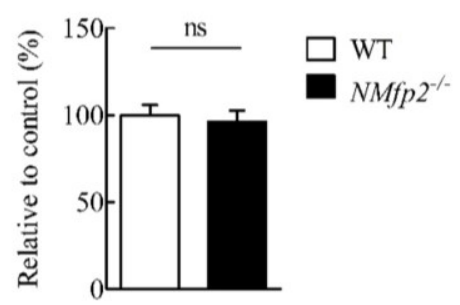

Figure 8: MFP2 deficiency leads to aberrant PC afferent innervation. Representative double stainings of calbindin withVGluT2 (A, D), VGluT1 (B, E) and VGaT (C, F) in 4-week-old Nestin-Mfp2${ }^{\prime}$ and WT mice. Significant decrease of CF (A, D, G) and PF (B, E, J) synapses in the ML of Nestin$M f p 2^{-/-}$mice. (H) CF extension is significantly reduced in Nestin-Mfp $2^{-/-}$mice while (I) more VGluT2 positive synapses are present on the PC soma. $(\mathrm{C}, \mathrm{F}, \mathrm{K}) \mathrm{PC}$ inhibitory innervation is unaltered. $\mathrm{N}=4$ mice per group. Results are displayed as mean \pm SEM. NS: not significant; ${ }^{*} \mathrm{p}<0.05 ;{ }^{*} \mathrm{p}<0.01$; $* * * \mathrm{p}<0.001$. Scale bars: $50 \mu \mathrm{m}$. 
A

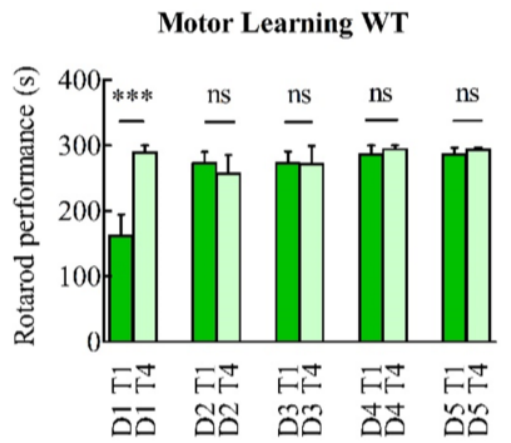

B

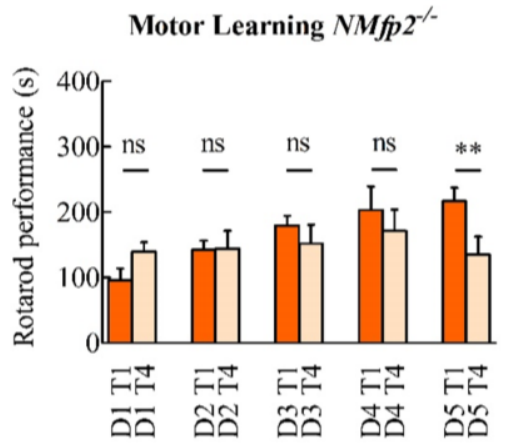

Supplementary figure 1: Motor learning in 4-week-old Nestin-Mfp $2^{-/}$and WT mice. (A, B) Rotarod performance on the first and last trials per day during 5 consecutive days. Nestin-Mfp $2^{-/}$mice perform less on the rotating rod during the last trial of the day. D1 - D5: day 1 - day 5; T1 - T4: trial 1 - trial 4 . $\mathrm{N}=6$ mice per group. Results are displayed as mean \pm SEM. NS: not significant; ${ }^{* *} \mathrm{p}<0.01 ;{ }^{* * *} \mathrm{p}<0.001$. 
A

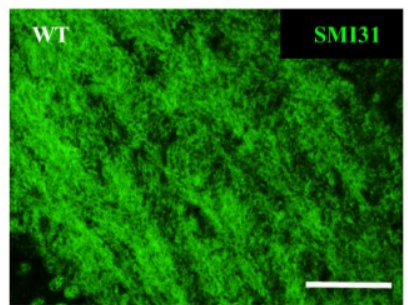

D

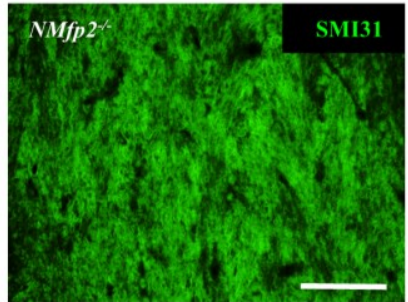

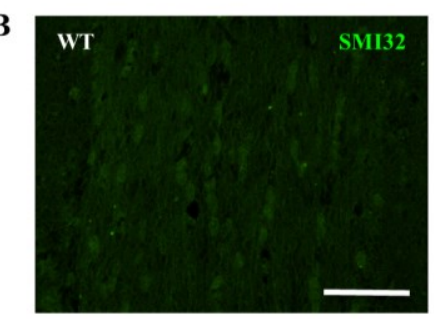

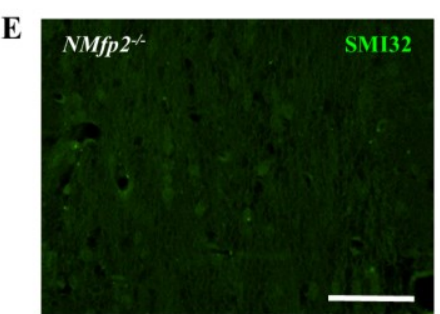

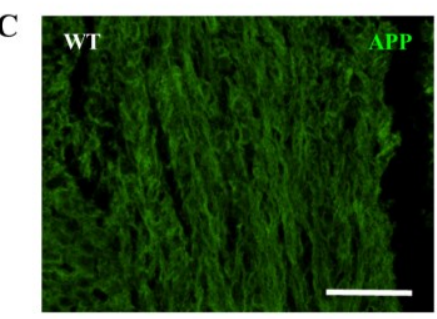

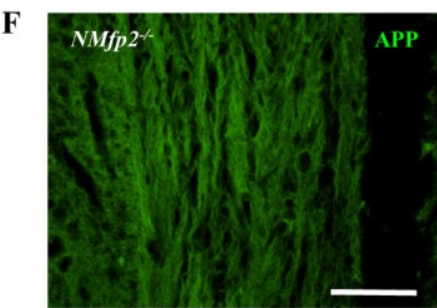

Supplementary figure 2: Absence of axonal damage in the corpus callosum of 4-weeks-old Nestin$\boldsymbol{M f p} \mathbf{2}^{-/}$mice. (A, D) SMI31 staining shows heavily phosphorylated neurofilaments in axons in the corpus callosum of WT and Nestin-Mfp $2^{--}$mice. The absence of SMI32 staining (B, E) and APP accumulation $(\mathrm{C}, \mathrm{F})$ points to uninterrupted axonal transport in both genotypes. Scale bars: $100 \mu \mathrm{m}$. 
Supplementary table 1: Electrophysiological parameters of WT and Nestin-Mfp ${ }^{-/}$PCs

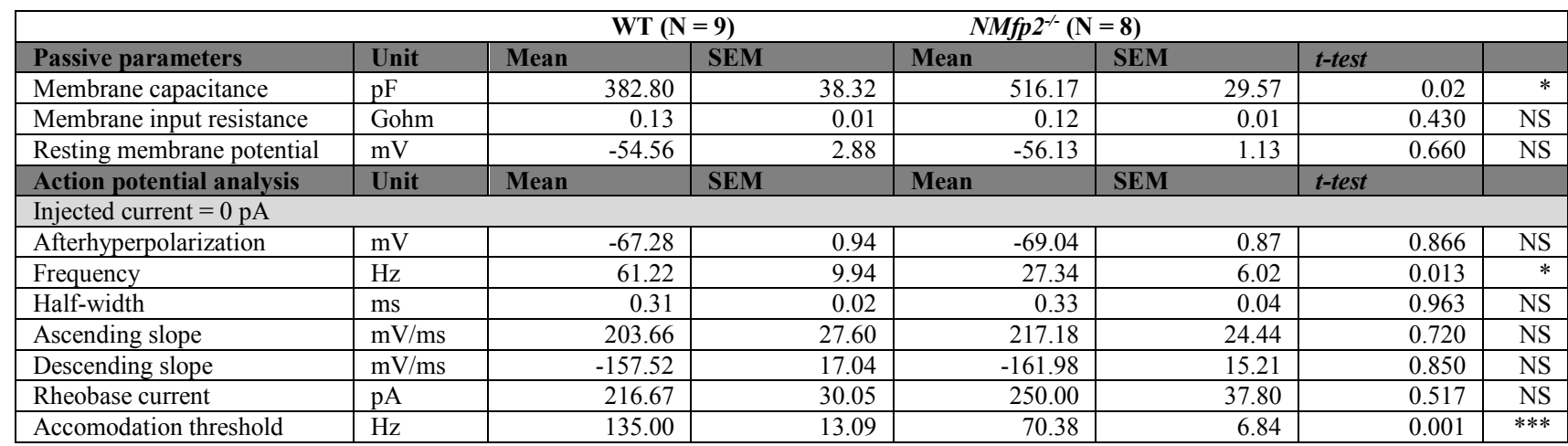




\section{AKNOWLEDGEMENTS}

The authors wish to thank Benno Das, Lies Pauwels, Laurie Lambot and Laetitia Cuvelier for their excellent assistance and Prof. L. Moons for providing the VGaT antibodies. This work was funded by grants from Fonds Wetenschappelijk Onderzoek Vlaanderen (G.0760.09 and G.0675.12) and Bijzonder Onderzoeksfonds KU Leuven (OT08/40 and OT12/79), FMRE-Belgium, FRS-FNRS (Belgium), Interuniversity Attraction Pole (IUAP-P7/10) from Belgian Federal Scientific Affairs. Pedro Brites is a FCT Investigator. 


\section{REFERENCES}

Baarine, M., et al., 2015. ABCD1 deletion-induced mitochondrial dysfunction is corrected by SAHA: implication for adrenoleukodystrophy. J Neurochem. 133, 380-96.

Baes, M., et al., 2000. Inactivation of the peroxisomal multifunctional protein-2 in mice impedes the degradation of not only 2-methyl-branched fatty acids and bile acid intermediates but also of very long chain fatty acids. J Biol Chem. 275, 16329-36.

Barkovich, A. J., Peck, W. W., 1997. MR of Zellweger syndrome. AJNR Am J Neuroradiol. 18, 1163-70.

Bergeron, Y., et al., 2014. mTOR signaling contributes to motor skill learning in mice. Front Mol Neurosci. 7, 26.

Brooks, S. P., Dunnett, S. B., 2009. Tests to assess motor phenotype in mice: a user's guide. Nat Rev Neurosci. 10, 519-29.

Bureau, G., et al., 2010. Intrastriatal inhibition of extracellular signal-regulated kinases impaired the consolidation phase of motor skill learning. Neurobiol Learn Mem. 94, 107-15.

Chen, X. R., et al., 2013. Mature Purkinje cells require the retinoic acid-related orphan receptor-alpha (RORalpha) to maintain climbing fiber mono-innervation and other adult characteristics. $J$ Neurosci. 33, 9546-62.

da Silva, T. F., et al., 2014. Peripheral nervous system plasmalogens regulate Schwann cell differentiation and myelination. J Clin Invest. 124, 2560-70.

De Munter, S., et al., 2015. Peroxisomal Disorders: A Review on Cerebellar Pathologies. Brain Pathol. $25,663-78$.

Dirkx, R., et al., 2005. Absence of peroxisomes in hepatocytes causes mitochondrial and ER abnormalities. Hepatology. 41, 868-878.

Faust, P. L., 2003. Abnormal cerebellar histogenesis in PEX2 Zellweger mice reflects multiple neuronal defects induced by peroxisome deficiency. J Comp Neurol. 461, 394-413.

Faust, P. L., et al., 2005. Peroxisome biogenesis disorders: the role of peroxisomes and metabolic dysfunction in developing brain. J Inherit Metab Dis. 28, 369-83.

Ferdinandusse, S., et al., 2006. Clinical and biochemical spectrum of D-bifunctional protein deficiency. Ann Neurol. 59, 92-104.

Ferdinandusse, S., et al., 2008. Ataxia with loss of Purkinje cells in a mouse model for Refsum disease. Proc Natl Acad Sci U S A. 105, 17712-7.

Gentet, L. J., et al., 2000. Direct measurement of specific membrane capacitance in neurons. Biophys J. 79, 314-20.

Gootjes, J., et al., 2004. Reinvestigation of trihydroxycholestanoic acidemia reveals a peroxisome biogenesis disorder. Neurology. 62, 2077-81.

Harno, E., et al., 2013. Metabolic pitfalls of CNS Cre-based technology. Cell Metab. 18, 21-8.

Hashimoto, K., Kano, M., 2005. Postnatal development and synapse elimination of climbing fiber to Purkinje cell projection in the cerebellum. Neurosci Res. 53, 221-8.

Herson, P. S., et al., 2003. A mouse model of episodic ataxia type-1. Nat Neurosci. 6, 378-83.

Hourez, R., et al., 2011. Aminopyridines correct early dysfunction and delay neurodegeneration in a mouse model of spinocerebellar ataxia type 1. J Neurosci. 31, 11795-807.

Hulshagen, L., et al., 2008. Absence of functional peroxisomes from mouse CNS causes dysmyelination and axon degeneration. Journal of neuroscience. 28, 4015-4027.

Itoh, M., et al., 2000. Developmental and pathological expression of peroxisomal enzymes : their relationship of D-bifunctional protein deficiency and Zellweger syndrome. Brain Research. 858, 40-47.

Itoh, M., et al., 1999. A novel peroxisomal enzyme, D-3-hydroxyacyl-CoA dehydratase/D-3hydroxyacyl-CoA dehydrogenase bifunctional protein : its expression in the developing human brain. Microscopy Research and Technique. 45, 383-388.

Ko, D. C., et al., 2005. Cell-autonomous death of cerebellar purkinje neurons with autophagy in Niemann-Pick type C disease. PLoS Genet. 1, 81-95. 
Komatsu, M., et al., 2007. Essential role for autophagy protein Atg7 in the maintenance of axonal homeostasis and the prevention of axonal degeneration. Proc Natl Acad Sci U S A. 104, 1448994.

Kruska, N., et al., 2015. Astrocytes and mitochondria from adrenoleukodystrophy protein (ABCD1)deficient mice reveal that the adrenoleukodystrophy-associated very long-chain fatty acids target several cellular energy-dependent functions. Biochim Biophys Acta. 1852, 925-36.

Krysko, O., et al., 2007. Neocortical and cerebellar developmental abnormalities in conditions of selective elimination of peroxisomes from brain or from liver. J Neurosci Res. 85, 58-72.

Kurihara, H., et al., 1997. Impaired parallel fiber-->Purkinje cell synapse stabilization during cerebellar development of mutant mice lacking the glutamate receptor delta2 subunit. J Neurosci. 17, 9613-23.

Lieber, D. S., et al., 2014. Next generation sequencing with copy number variant detection expands the phenotypic spectrum of HSD17B4-deficiency. BMC Med Genet. 15, 30.

Lines, M. A., et al., 2014. Peroxisomal D-bifunctional protein deficiency: three adults diagnosed by whole-exome sequencing. Neurology. 82, 963-8.

Llano, I., et al., 1991. Synaptic- and agonist-induced excitatory currents of Purkinje cells in rat cerebellar slices. J Physiol. 434, 183-213.

Lorenzetto, E., et al., 2009. Genetic perturbation of postsynaptic activity regulates synapse elimination in developing cerebellum. Proc Natl Acad Sci U S A. 106, 16475-80.

Mahad, D., et al., 2008. Review: Mitochondria and disease progression in multiple sclerosis. Neuropathol Appl Neurobiol. 34, 577-89.

Mahad, D. J., et al., 2009. Mitochondrial changes within axons in multiple sclerosis. Brain. 132, 116174.

McMillan, H. J., et al., 2012. Specific combination of compound heterozygous mutations in 17betahydroxysteroid dehydrogenase type 4 (HSD17B4) defines a new subtype of D-bifunctional protein deficiency. Orphanet J Rare Dis. 7, 90.

Mignarri, A., et al., 2012. Zellweger Spectrum Disorder with Mild Phenotype Caused by PEX2 Gene Mutations. JIMD Rep. 6, 43-6.

Milnerwood, A. J., et al., 2010. Early increase in extrasynaptic NMDA receptor signaling and expression contributes to phenotype onset in Huntington's disease mice. Neuron. 65, 178-90.

Moller, G., et al., 1999. Characterization of the HSD17B4 gene: D-specific multifunctional protein 2/17beta-hydroxysteroid dehydrogenase IV. J.Steroid Biochem.Mol.Biol. 69, 441-446.

Muller, C. C., et al., 2011. PEX13 deficiency in mouse brain as a model of Zellweger syndrome: abnormal cerebellum formation, reactive gliosis and oxidative stress. Dis Model Mech. 4, 104-19.

Nguyen-Vu, T. D., et al., 2013. Cerebellar Purkinje cell activity drives motor learning. Nat Neurosci. 16, 1734-6.

Petrinovic, M. M., et al., 2013. Neuronal Nogo-A negatively regulates dendritic morphology and synaptic transmission in the cerebellum. Proc Natl Acad Sci U S A. 110, 1083-8.

Pierce, S. B., et al., 2010. Mutations in the DBP-deficiency protein HSD17B4 cause ovarian dysgenesis, hearing loss, and ataxia of Perrault Syndrome. Am J Hum Genet. 87, 282-8.

Raas-Rothschild, A., et al., 2002. A PEX6-defective peroxisomal biogenesis disorder with severe phenotype in an infant, versus mild phenotype resembling Usher syndrome in the affected parents. Am J Hum Genet. 70, 1062-8.

Ramer, M. S., et al., 2010. A new organellar complex in rat sympathetic neurons. PLoS One. 5, e10872.

Redondo, J., et al., 2015. Purkinje Cell Pathology and Loss in Multiple Sclerosis Cerebellum. Brain Pathol. 25, 692-700.

Regal, L., et al., 2010. Mutations in PEX10 are a cause of autosomal recessive ataxia. Ann Neurol. 68, 259-63.

Sevin, C., et al., 2011. Autosomal recessive cerebellar ataxia caused by mutations in the PEX2 gene. Orphanet J Rare Dis. 6, 8.

Tavazoie, S. F., et al., 2005. Regulation of neuronal morphology and function by the tumor suppressors Tsc1 and Tsc2. Nat Neurosci. 8, 1727-34. 
Teigler, A., et al., 2009. Defects in myelination, paranode organization and Purkinje cell innervation in the ether lipid-deficient mouse cerebellum. Hum Mol Genet. 18, 1897-908.

Vandeputte, C., et al., 2010. Automated quantitative gait analysis in animal models of movement disorders. BMC.Neurosci. 11, 92.

Verheijden, S., et al., 2013. Peroxisomal multifunctional protein-2 deficiency causes neuroinflammation and degeneration of Purkinje cells independent of very long chain fatty acid accumulation. Neurobiol Dis. 58, 258-69.

Walkley, S. U., et al., 2010. Lysosomal compromise and brain dysfunction: examining the role of neuroaxonal dystrophy. Biochem Soc Trans. 38, 1436-41.

Wang, Q. J., et al., 2006. Induction of autophagy in axonal dystrophy and degeneration. J Neurosci. 26, 8057-68.

Zala, D., et al., 2013. Vesicular glycolysis provides on-board energy for fast axonal transport. Cell. 152, 479-91. 